\title{
Electron impact ionization of hydrogen-like molybdenum ions
}

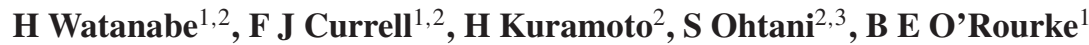 \\ and $\mathrm{X} M$ Tong ${ }^{2,4}$ \\ ${ }^{1}$ Department of Physics, Queen's University Belfast, Belfast BT7 1NN, UK \\ 2 Cold Trapped Ion Project, ICORP, JST, Saitama 332-0012, Japan \\ ${ }^{3}$ University of Electro-Communications, Tokyo 182-8585, Japan \\ ${ }^{4}$ Physics Department, Kansas State University, Manhattan, KS 66506, USA
}

Received 28 June 2002, in final form 1 October 2002

Published 4 December 2002

Online at stacks.iop.org/JPhysB/35/5095

\begin{abstract}
The electron impact ionization cross sections of hydrogen-like molybdenum ions were measured with an electron beam ion trap at the electron energies of 49.4, 64.4 and $79.6 \mathrm{keV}$. The results are $2.82(22) \times 10^{-23}, 3.13(29) \times 10^{-23}$ and $3.23(51) \times 10^{-23} \mathrm{~cm}^{2}$, respectively. These results are compared with the experimental results measured previously. The agreement with the results obtained with scaling formulae is also discussed.
\end{abstract}

\section{Introduction}

Electron impact ionization (EI) is one of the primary processes in plasmas and its cross section is of overriding importance to model fusion plasmas and astrophysical plasmas. Although EI cross sections have been measured for various elements and various incoming electron energies, the measurements for highly charged ions, especially for hydrogen-like ions, were performed quite recently [1-3]. For low atomic number $(Z)$ elements, crossed beam methods have been used $[1,3,4]$. However for high $Z$ elements, such methods have not been used so far, since it is difficult to produce enough ions. Electron beam ion traps (EBITs) have been developed to make highly charged ions. Marrs et al [2] measured EI cross sections of H-like high- $Z$ ions by considering the charge balance in the trap. The relativistic effects and the effect of Breit interaction which is the lowest-order QED effect were observed in their results.

Many scaling formulae for EI cross sections have been proposed due to the importance of the process for plasma modelling [1, 3, 5-10]. The semi-empirical formula proposed by Lotz [5] is well known and has been used frequently. In spite of its simplicity, it can reproduce experimental results quite well. Recently, the universal shape function which shows the energy dependence of non-relativistic H-like EI cross sections was proposed by Aichele et al [3]. They constructed a function so that it approached the Bethe limit at high energy and followed the Wannier law [11] near the ionization threshold. They expressed the function by using 
the maximum value of the cross section and the electron energy measured from the ionization threshold where the maximum occurs. On the other hand, scaling formulae have been obtained by fitting appropriate functions to theoretical results. Recently, Fontes et al [10] obtained a scaling formula with the relativistic effects and the effect of Breit interaction considered, by fitting a function to their calculations. In the case of high- $Z$ elements and high electron energies it is known that the relativistic effects and the effect of Breit interaction become more important [12]. It is essential to verify the validity of these formulae for various elements and various electron energies.

In this study we have measured the EI cross section of H-like molybdenum ions at three different energies. The cross section of H-like Mo ions was also measured by Marrs et al [2]. We verify and complement their results through our measurements. In the range of $Z$ around 42 the relativistic effects and the effect of Breit interaction start to become more important [12]. In the case of $\mathrm{H}$-like Fe at $Z=26$, the cross sections can be expressed with the semi-empirical formulae proposed by Lotz [5] and by Aichele et al [3]. We will compare our results with those obtained with these scaling formulae and test their validity. There are only a few reported measurements of the EI cross section of H-like ions with $Z$ from 20 to 50 [2, 13]. In fusion plasmas it is well known that many kinds of impurity ions exist due to the sputtering of the wall materials. The understanding of the atomic processes of the elements with industrial importance such as Mo is also essential to control the impurity ions in plasmas.

\section{Experimental details}

The measurements were performed with the use of the Tokyo-EBIT [14] with a technique which is an extension of the method used by Marrs et al [2]. The charge balance between bare and $\mathrm{H}$-like ions was determined by the ionization of $\mathrm{H}$-like ions by electron impact, the recombination of bare ions by radiative electron capture and charge exchange collisions with neutrals, and the escape of bare and H-like ions from the trap. In the EBIT to achieve the longer trapping time, the ions are cooled with the method called evaporative cooling [15]. In evaporative cooling, low- $Z$ neutrals are introduced into the trap. Once ionized, the low- $Z$ species take heat from the trapped ions through ion-ion collisions and escape from the trap. As a consequence the escape from the trap of the more highly charged ions under study can be ignored, although, instead, the recombination by the charge exchange collisions has an effect on the charge balance in the trap.

When equilibrium is achieved, the charge balance in the trap can be written

$$
f_{H} \frac{J_{e}}{e} N_{H} \sigma_{H}^{E I}=f_{B} \frac{J_{e}}{e} N_{B} \sigma_{B}^{\sum R R}+N_{0} N_{B}\left\langle v \sigma_{B}^{C X}\right\rangle
$$

where $N_{B}$ and $N_{H}$ are the number densities of bare and H-like ions, $J_{e}$ is the electron beam current density, and $N_{0}$ the number density of neutral atoms. $f_{B}$ and $f_{H}$ are the overlap factors of the ion cloud and the electron beam of bare and H-like ions, respectively. Their values are approximately equal and close to 1.0 because of the shallow trapping used. $e$ is the elementary charge. $\sigma_{H}^{E I}$ is the EI cross section of $\mathrm{H}$-like ions, $\sigma_{B}^{\sum_{B} R R}$ the total radiative recombination (RR) cross section of bare ions and $\left\langle v \sigma_{B}^{C X}\right\rangle$ the charge exchange rate coefficient of bare ions with neutrals.

The intensity of the $n=1 \mathrm{RR}$ x-rays observed at right angles with respect to the electron beam axis, $N_{h v}$, is related to the number of the trapped ions, $N$, as $N_{h v s}=$ $f_{s}\left(I_{e} / e r_{b}^{2}\right) N_{s}\left(\mathrm{~d} \sigma_{s}^{R R=1} / \mathrm{d} \Omega\right) \mathrm{d} \Omega$, for species $s$ (either bare, B or H-like, $\left.\mathrm{H}\right) . I_{e}$ is the electron beam current and $r_{b}$ the beam radius. $\mathrm{d} \sigma^{R R=1} / \mathrm{d} \Omega$ is the differential $n=1$ RR cross sections in the direction of the observation. $\mathrm{d} \Omega$ is the solid angle of the observation. 
Therefore the intensity ratio of the $n=1 \mathrm{RR} x$-rays is

$$
\frac{N_{h v_{H}}}{N_{h v_{B}}}=\frac{\sigma_{B}^{\sum R R} \frac{\mathrm{d} \sigma_{H}^{R R=1}}{\mathrm{~d} \Omega}}{\sigma_{H}^{E I} \frac{\mathrm{d} \sigma_{B}^{R R=1}}{\mathrm{~d} \Omega}}\left(1+a\left\langle v \sigma_{B}^{C X}\right\rangle \frac{N_{0}}{I_{e}}\right)
$$

where $a=e r_{b}^{2} /\left(f_{B} \sigma_{B}^{\sum R R}\right)$, which is approximately constant through the experiment.

In the limit $N_{0} \rightarrow 0$ or $I_{e} \rightarrow \infty$, the EI cross section can be expressed as

$$
\sigma_{H}^{E I}=\frac{N_{h v_{B}}}{N_{h v_{H}}} \frac{\sigma_{B}^{\sum R R} \frac{\mathrm{d} \sigma_{H}^{R R=1}}{\mathrm{~d} \Omega}}{\frac{\mathrm{d} \sigma_{B}^{R R=1}}{\mathrm{~d} \Omega}}
$$

without using $a\left\langle v \sigma_{B}^{C X}\right\rangle$ which is difficult to obtain. We can obtain the impact ionization cross section, having the intensity ratio of RR in either limit $N_{0} \rightarrow 0$ or $I_{e} \rightarrow \infty$, and the total and differential RR cross sections. This intensity ratio is obtained by experiments. The theoretical RR cross sections can be used, since they can be calculated with high accuracy [16].

Actually the measurements at $N_{0}=0$ or $I_{e}=\infty$ are not possible. Marrs et al [2] obtained the intensity ratios at $N_{0}=0$ by extrapolation, using the results obtained at various neutral gas densities. O'Rourke et al [13] measured the intensity ratios at various neutral gas densities and also at various electron beam currents. They extrapolated the ratio at $N_{0}=0$ and $I_{e}=\infty$ so that the extrapolated values took the same value for both the measurements. We have performed the experiment by changing the beam current to extrapolate the ratio at $I_{e}=\infty$.

Kuramoto et al [17] showed that the electron beam radius of the EBIT was almost independent of the electron beam current. The pressure in the gas injector had a measured variation of typically less than $2 \%$ throughout the experimental run. Since the injected gas pressure is related to this pressure by an unknown geometrical constant, it is safe to assume that the injected pressure in the trap also had a similar variation. Furthermore, since the electron beam was thin enough not to hit the trap electrodes, the thermal environment of the trap would have been constant throughout the experiment. The overlap factor is almost constant with value close to 1 for the present range of electron energy, current and axial trapping depth. The ion velocity is also almost constant, since the axial trapping depth was shallow, being $100 \mathrm{~V}$ throughout the experiment. Thus the ratio is inversely proportional to the electron beam current as shown in equation (2).

It should be noted that, in the method used in this study, it is not necessary to use the pressure value in the trap, which could not be measured practically. In fact Marrs et al [2] and O'Rourke et al [13] used the pressure values at the gas injector, assuming that they were proportional to the value in the trap. Furthermore, sufficient coolant gas can be introduced into the trap every time, resulting in higher $\mathrm{x}$-ray intensity.

We have measured the cross sections at the electron energy of 49.4, 64.4 and $79.6 \mathrm{keV}$ (2.01, 2.62, 3.24 times of the ionization energy of H-like Mo ions). Source Mo ions were introduced into the EBIT with a metal vapour vacuum arc (MEVVA) ion source installed at the top of the EBIT. When the MEVVA was fired, the potential applied to the upper drift tube (called DT3) was lowered. Just after this, the potential was applied to the DT3 again and the trapping started. The X-rays were observed with a Ge detector, which was calibrated with radioisotopes ${ }^{241} \mathrm{Am},{ }^{109} \mathrm{Cd}$ and ${ }^{57} \mathrm{Co}$. The observed $\mathrm{x}$-ray signal was stored in a computer through a multiparameter data acquisition system [18]. The time information for X-ray detection was stored simultaneously. The signal to raise the DT3 potential was used as the start signal of the time information. The trapping period was $20 \mathrm{~s}$. The trapped ions were dumped every $20 \mathrm{~s}$ to avoid accumulation of unwanted ions such as Ba originating from the cathode of the electron gun. The inject, trap, measure, dump cycle was repeated many times 
for each set of experimental parameters to improve the statistical quality of the data. Ne gas was introduced into the trap for evaporative cooling. The measurements were performed at the electron beam currents of 125, 150, 170, 250 and $300 \mathrm{~mA}$ for $49.4 \mathrm{keV}, 125,150,170$, 200, 250 and $300 \mathrm{~mA}$ for $64.4 \mathrm{keV}$, and 85, 110, 150, 225 and $300 \mathrm{~mA}$ for $79.6 \mathrm{keV}$.

\section{Results and discussion}

Equation (1) only holds when the trapped ions reach equilibrium. In fact for bare Mo ions a few seconds may be required to reach equilibrium. The intensity variation of RR $\mathrm{x}$-rays with respect to time was checked for both the bare and $\mathrm{H}$-like ions to confirm the equilibrium condition. The spectra binned in one-second intervals from the start of the trapping were composed with the use of the time information of x-ray detection. The heights of the lines were obtained by the fitting of Gaussian functions shown below:

$$
f(x)=A_{H} \exp \left[-\frac{\left(x-E_{H}-E_{e}\right)^{2}}{\sigma}\right]+A_{B} \exp \left[-\frac{\left(x-E_{B}-E_{e}\right)^{2}}{\sigma}\right]+C
$$

$A_{B}$ and $A_{H}$ are the height of RR lines of bare and H-like ions, respectively. $C$ is a constant. $E_{B}$ and $E_{H}$ are the ionization energies of H-like and He-like Mo ions, which are 24.57 and $23.81 \mathrm{keV}$, respectively [19]. $E_{e}$ is the electron energy. $\sigma$ is related to the resolution of the detector. The same value is used for the bare and $\mathrm{H}$-like lines.

The $\mathrm{x}$-ray counts follow a Poisson distribution. The $\mathrm{x}$-ray counts of the spectra measured during every one second were low. In such cases, the least-square fitting may not give the correct values, since it is based on the fact that the measured values follow a normal distribution. In this study, 'Poisson fitting' was used [20]. In Poisson fitting the fitting parameters which are the solution of the following simultaneous equations are derived.

$$
\sum_{i=1}^{n} \frac{y_{i}-f\left(x_{i}\right)}{f\left(x_{i}\right)} \frac{\partial f\left(x_{i}\right)}{\partial a_{k}}=0 \quad(k=1, \ldots m)
$$

where $\left(x_{i}, y_{i}\right)$ are the experimental values, $f(x)$ the fitting function and $a_{k}$ the fitting parameters.

Figure 1 shows the intensity variations of the RR x-rays with respect to time for the measurement with $64.4 \mathrm{keV} 170 \mathrm{~mA}$ electron beam. As shown in the figure, H-like ions reach the equilibrium within $3 \mathrm{~s}$ after trapping has started, while bare ions take about $7 \mathrm{~s}$. In the case of bare ions the intensity gradually decreases from $17 \mathrm{~s}$, which means that ions start to escape from the trap from that time. This may be due to the slow accumulation of highly charged heavy ions such as Ba. Once a sufficient amount of these ions accumulates, Mo ions act as a coolant for these ions and escape from the trap preferentially. We consider that the equilibrium was achieved between 7 and $16 \mathrm{~s}$ and the escape of bare ions was negligible during this period. The spectra measured during this period were summed and, then, the spectrum obtained was fitted with the function in equation (4). The result is shown in figure 2. With this procedure we obtained the heights of RR lines of bare and H-like ions and also the electron energy. We use the fitted result as the electron energy, based on the fact that the RR X-ray energy is the sum of the ionization energy and the electron energy. The x-ray energy was precisely calibrated and the ionization energy was known [19]. The electron energy scale was derived from the fit to the measured $\mathrm{x}$-ray spectrum in this way, rather than from the difference of the potential of the cathode and trap, as determined by reading power supplies. This procedure was adopted to remove the systematic error in the electron energy scale due to the space charge of the electron beam. 

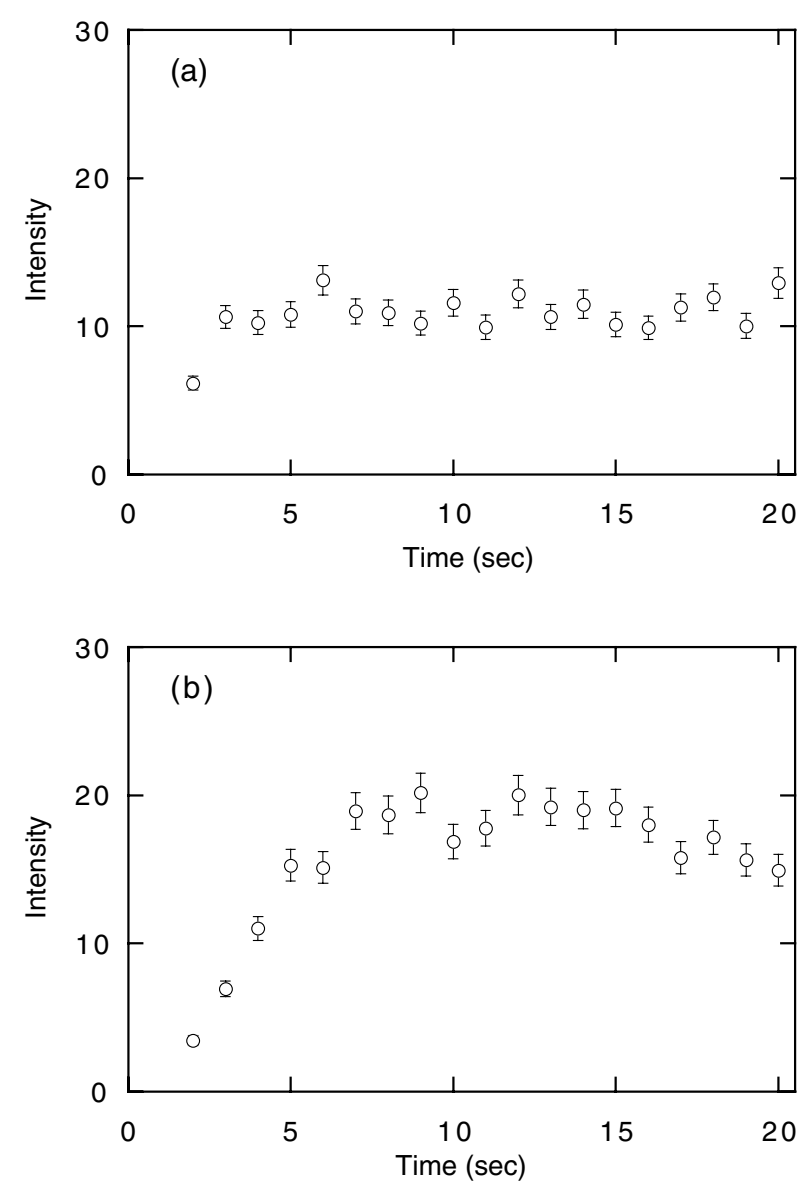

Figure 1. The intensity variations of RR x-ray for H-like ions (a) and for bare ions (b) with respect to time, measured with the $64.4 \mathrm{keV} 170 \mathrm{~mA}$ electron beam. H-like ions reach equilibrium within $3 \mathrm{~s}$ after the trapping started. For bare ions equilibrium is achieved between 7 and $16 \mathrm{~s}$.

Table 1. The ionization cross sections for H-like Mo ions. The theoretical total and differential RR cross sections are also summarized. The second column is the electron energy in ionization threshold units. The total RR cross sections and the ionization cross sections are in units of $10^{-24} \mathrm{~cm}^{2}$. The differential cross sections are in units of $10^{-24} \mathrm{~cm}^{2} \mathrm{Sr}^{-1}$.

\begin{tabular}{llllll}
\hline$E_{e}(\mathrm{keV})$ & $E_{e}$ & $\frac{\mathrm{d} \sigma_{H}^{R R=1}}{\mathrm{~d} \Omega}$ & $\frac{\mathrm{d} \sigma_{B}^{R R=1}}{\mathrm{~d} \Omega}$ & $\sigma_{B} R R$ & $\sigma_{H}^{E I}$ \\
\hline 49.4 & 2.01 & 1.332 & 2.687 & 42.76 & $28.2 \pm 2.2$ \\
64.4 & 2.62 & 0.7670 & 1.545 & 26.87 & $31.3 \pm 2.9$ \\
79.6 & 3.24 & 0.4762 & 0.9575 & 18.31 & $32.3 \pm 5.1$ \\
\hline
\end{tabular}

Figure 3 shows the intensity ratio of the RR x-rays with respect to the inverse of the electron beam current. The errors in the figure originate from the counting statistics alone. As expected from equation (2), the ratios are linearly dependent on the inverse of the electron beam current. A linear function was fitted to obtain the intercept and its error. Using equation (3) we obtained the EI cross section. The obtained results are listed in table 1. 


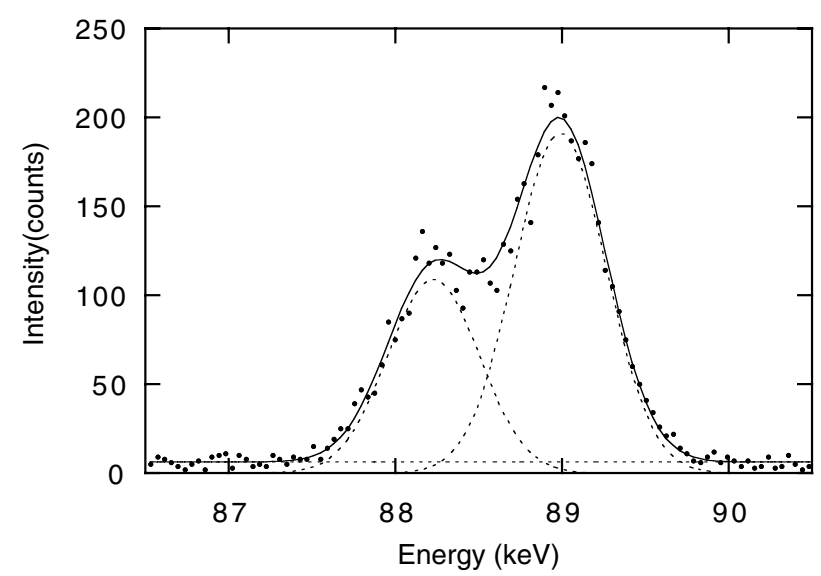

Figure 2. The RR x-ray spectrum measured with a $64.4 \mathrm{keV} 170 \mathrm{~mA}$ electron beam. The solid curve is the fitted result using equation (4) and the dotted curves are the components of the fit.

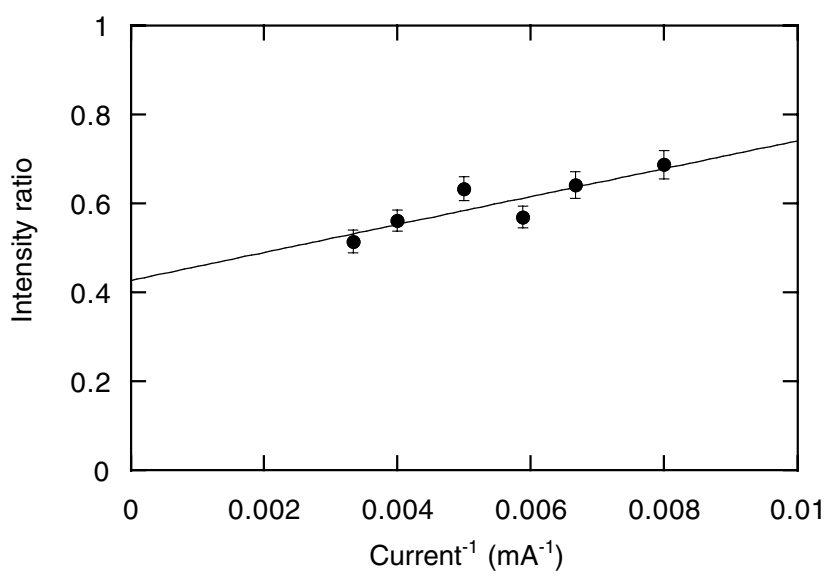

Figure 3. The intensity ratio of the RR $\mathrm{x}$-ray $\left(N_{h v_{H}} / N_{h v_{B}}\right)$ with respect to the inverse of the electron beam current $\left(1 / I_{e}\right)$. These measurements were performed at the $64.4 \mathrm{keV}$ electron beam energy. The solid line is the line fitted to the data. The errors shown on this figure come from the counting statistics alone.

In table 1 we also summarize the total RR cross sections and the differential $n=1 \mathrm{RR}$ cross sections, which we calculated for this study. These values were obtained from photoionization cross sections with the detailed balance principle. The photoionization cross sections and their angular dependences were calculated using the independent particle approximation [21-23] with an effective potential obtained with relativistic density functional theory [24] with an optimized effective potential and self-interaction correction method.

The EI cross sections of H-like Mo ions were measured previously by Marrs et al [2] with an EBIT. They used the method explained above, though they measured the RR X-rays, changing the pressure in the trap. Our results and those of Marrs et al [2] are shown in figure 4. Marrs et al [2] measured at $64.8 \mathrm{keV}$ and their result was $3.08(26) \times 10^{-23} \mathrm{~cm}^{2}$. We have also measured at $64.4 \mathrm{keV}$, which was $3.13(29) \times 10^{-23} \mathrm{~cm}^{2}$. Both the results at $65 \mathrm{keV}$ agree well with each other. The variation of the cross sections with respect to the electron energy seems 


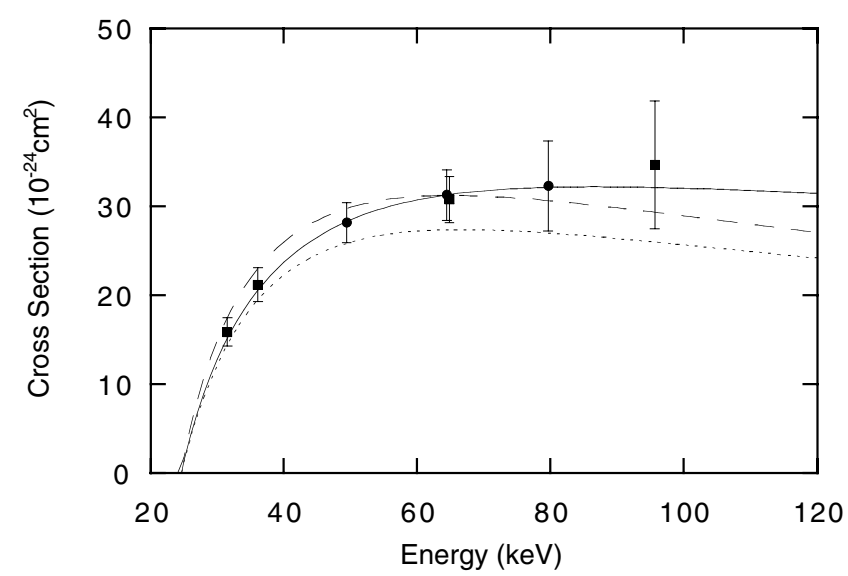

Figure 4. The electron EI cross sections of H-like Mo Ions. The circles are the present results and the squares are those obtained by Marrs et al [2]. The curves are calculated with the scaling formulae proposed by Lotz [5] (dotted curve), Aichele et al [3] (dashed curve) and Fontes et al [10] (solid curve).

consistent, as seen in the figure. The reliability of the ionization cross sections of H-like Mo ions becomes higher due to this consistency.

Simple expressions to show the EI cross sections have been developed by many researchers. The semi-empirical scaling formula proposed by Lotz [5] has been used and has the form for H-like ions

$$
\sigma_{H}^{E I}=a_{i} \frac{\ln \left(E_{e} / I\right)}{E_{e} I}, \quad a_{i}=4.5 \times 10^{-14} \mathrm{~cm}^{2}(\mathrm{eV})^{2}
$$

where $E_{e}$ is the electron energy and $I$ the ionization energy.

Recently, a universal shape function has been proposed [3, 25], which has a $1 / E_{e}$ dependence in the high energy limit and an $E_{e}-I$ dependence near the ionization threshold:

$$
\sigma_{H}^{E I}(x)=\sigma_{M}[\cosh (0.4 \ln x)]^{-1 / 0.4}
$$

where $x=\left(E_{e}-I\right) / E_{M} . \sigma_{M}$ is the maximum value of the cross section and $E_{M}$ the beam energy measured as the excess energy above threshold, where the maximum occurs. The expressions of $E_{M}(Z)$ and $\sigma_{M}(Z)$ with $Z$ derived using the existing experimental data are

$$
\begin{aligned}
& E_{M}(Z)=I_{H}\left[3.31+5.07(Z-1)+1.65(Z-1)^{2}\right] \\
& \sigma_{M}(Z)=\pi a_{0}^{2}\left(\frac{1.0278}{1.0975+Z-1}\right)^{4}
\end{aligned}
$$

where $I_{H}$ is the ionization energy of the hydrogen atom and $a_{0}$ the Bohr radius.

Fontes et al [10] performed theoretical calculation using the relativistic distorted wave method with the generalized Breit interaction included. They obtained a scaling formula by fitting a function to the result. Their result is

$\sigma_{H}^{E I}=\frac{\pi a_{0}^{2}}{I(\mathrm{Ryd})^{2}} Q^{\prime}[u, Z] F[Z]$

$F[Z]=\left[140+(Z / 20)^{3.2}\right] / 141$

$Q^{\prime}[u, Z]=\frac{1}{u}\left[1.13 \ln u+3.7059\left(1-\frac{1}{u}\right)^{2}+C[Z] u\left(1-\frac{1}{u}\right)^{4}\right.$ 


$$
\left.+\left(\frac{1.9527}{u^{2}}-\frac{0.28394}{u}\right)\left(1-\frac{1}{u}\right)\right]
$$

where $C[Z]$ is

$$
\begin{aligned}
& C[Z]=[(Z-20) / 50.5]^{1.11}+0.20594, \quad 20 \leqslant Z \leqslant 66 \\
& C[Z]=[(Z-20) / 53]^{0.73}+0.20594, \quad 67 \leqslant Z \leqslant 92
\end{aligned}
$$

or

$$
C[Z]=[(Z-20) / 55]^{0.92}+0.20594, \quad 20 \leqslant Z \leqslant 92 .
$$

$I$ (Ryd) is the ionization energy in Rydberg units and $u=E_{e} / I$.

In figure 4 the results calculated with these formulae are shown together with the experimental results. In the low-energy region, the results obtained with these formulae take almost the same values and coincide well with the experimental results. However, the result obtained with Lotz's formula obviously underestimates the cross section over $40 \mathrm{keV}$.

The formula of Aichele et al [3] has a similar tendency to that obtained by Fontes et al [10] until the cross section takes the maximum at $65 \mathrm{keV}$ and then decreases gradually in contrast to that of Fontes et al [10]. In the formula of Fontes et al [10] the new term $C$ was added to the fitting function which had been used for the fitting to obtain scaling formulae for the non-relativistic EI cross sections [6]. This term is to reproduce the near-constant behaviour at high energies to take into account the relativistic effects and the effect due to Breit interaction. Therefore, the result used by Fontes et al [10] shows a different tendency to that of Aichele et al [3]. In the range of the energy where the experiments were performed, the formulae of Fontes et al [10] and Aichele et al [3] agree with the experimental results within the experimental errors. To verify the high-energy behaviour of these formulae experiments with high accuracy are required at high energies.

It was demonstrated that the relativistic effects and the effect of Breit interaction cannot be neglected in the high-energy region with the increase of $Z$ [12]. Therefore, it is expected that the formula proposed by Aichele et al [3] could not reproduce the experimental results in such region, since their formula did not consider these effects. However, we can conclude that their formula can be used, at least for the elements with $Z \leqslant 42$ up to the energy $E_{M}$ given by their formula.

\section{Conclusion}

We have measured the EI cross section of H-like Mo ions with the Tokyo-EBIT. The cross section was obtained from the charge balance between bare and $\mathrm{H}$-like ions at equilibrium. A method similar to that employed by Marrs et al [2] was used, though their technique was extended by measuring the dependence of the intensity of RR x-rays on electron beam current to extrapolate away the effect of the charge exchange collision with neutrals. The results are $2.82(22) \times 10^{-23}, 3.13(29) \times 10^{-23}$ and $3.23(51) \times 10^{-23} \mathrm{~cm}^{2}$ for $49.4,64.4$ and $79.6 \mathrm{keV}$, respectively. These results agree well with the result obtained by Marrs et al [2]. The scaling formula proposed by Lotz [5] underestimates the measured cross sections, while those proposed by Aichele et al [3] and Fontes et al [10] agree within the range of the experimental errors. The semi-empirical scaling formula proposed by Aichele et al [3] can reproduce the EI cross sections, at least for the elements with $Z \leqslant 42$ below the energy $E_{M}$ given by their formula. The scaling formula proposed by Fontes et al [10], which takes into account the relativistic effects and the effect of Breit interaction, can reproduce the results well over the whole energy range, although more measurements such as those presented here are required to provide a more stringent test. 


\section{Acknowledgments}

This work was performed under the auspices of the International Cooperative Research Project (ICORP) of the Japan Science and Technology Corporation. We are grateful to the Royal Society and Great Britain Sasakawa Foundation for additional funding which made the travel associated with this collaboration possible. HW is indebted to the Japan Society for the Promotion of Science for the support of the JSPS overseas research fellowship.

\section{References}

[1] Tinschert K, Müller A, Hofmann G, Huber K, Becker R, Gregory D C and Salzborn E 1989 J. Phys. B: At. Mol. Opt. Phys. 22531

[2] Marrs R E, Elliott S R and Scofield J H 1997 Phys. Rev. A 561338

[3] Aichele K, Hartenfeller U, Hathiramani D, Hofmann G, Schäfer V, Steidl M, Stenke M, Salzborn E, Pattard T and Rost J M 1998 J. Phys. B: At. Mol. Opt. Phys. 312369

[4] Dolder K T, Harrison M F A and Thonemann P C 1961 Proc. R. Soc. A 264367

[5] Lotz W 1968 Z. Phys. 216241

[6] Golden L B and Sampson D H 1977 J. Phys. B: At. Mol. Phys. 102229

[7] Sampson D H and Golden L B 1978 J. Phys. B: At. Mol. Phys. 11541

[8] Younger S M 1981 J. Quant. Spectrosc. Radiat. Transfer 26329

[9] Deutsch H, Becker K and Märk T D 1995 Int. J. Mass Spectrom. Ion Process. 151207

[10] Fontes C J, Sampson D H and Zhang H L 1999 Phys. Rev. A 591329

[11] Wannier G H 1953 Phys. Rev. 90817

[12] Moores D L and Reed K J 1995 Nucl. Instrum. Methods B 98122

[13] O'Rourke B, Currell F J, Kuramoto H, Li Y M, Ohtani S, Tong X M and Watanabe H 2001 J. Phys. B: At. Mol. Opt. Phys. 344003

[14] Currell F J et al 1996 J. Phys. Soc. Japan 653186

[15] Schneider M B, Levine M A, Bennett C L, Henderson J R, Knapp D A and Marrs R E 1989 Int. Symp. on Electron Beam Ion Sources and their Applications (AIP Conf. Proc. vol 188) ed A Hershcovitch (New York: American Institute of Physics) p 158

[16] Saloman E B, Hubbell J H and Scofield J H 1988 At. Data Nucl. Data Tables 381

[17] Kuramoto H, Kinugawa T, Watanabe H, Yamada C, Ohtani S, Yamada I and Currell F J 2002 Rev. Sci. Instrum. 7342

[18] Currell F J et al 1997 Phys. Scr. T 73371

[19] Shirai T, Sugar J, Musgrove A and Wiese W L 2000 Spectral Data For Highly Ionized Atoms: Ti, V, Cr, Mn, Fe, $\mathrm{Co}, \mathrm{Ni}, \mathrm{Cu}, \mathrm{Kr}$, and $\mathrm{Mo}$ (New York: American Institute of Physics) p 485

[20] Awaya T 1991 DETA KAISEKI: Data Analysis (Tokyo: Japan Scientific Societies Press) pp 179-209 (in Japanese)

[21] Tseng H K, Pratt R H, Yu S and Ron A 1978 Phys. Rev. A 171061

[22] Kim Y S, Pratt R H, Ron A and Tseng H K 1980 Phys. Rev. A 22567

[23] Scofield J H 1989 Phys. Rev. A 403045

[24] Tong X M and Chu S I 1998 Phys. Rev. A 57855

[25] Rost J M and Pattard T 1997 Phys. Rev. A 55 R5 